\title{
NLRP3 inflammasome mediates crystal-induced joint inflammation and dysfunction
}

Leukotriene B4 (LTB4) drives NLRP3 inflammasome activation in response to monosodium urate (MSU) crystals-ultimately contributing to joint inflammation and dysfunction - in a mouse model of gout. The findings, published in Arthritis \& Rheumatism, show that "LTB4 is important very early on in the context of MSU-induced inflammation, which opens up the possibility that targeting this pathway might be relevant in patients with gout," according to author Mauro Teixeira.

A definitive diagnosis of gout is confirmed when MSU crystals are identified in the synovial fluid, but exactly how these crystals affect the joint is unclear. "Existing evidence indicates that the NLRP3 inflammasome 'senses' MSU crystals and that IL- $1 \beta$ is involved, says Teixeira.

To determine just how MSU crystals influence joint function, the researchers

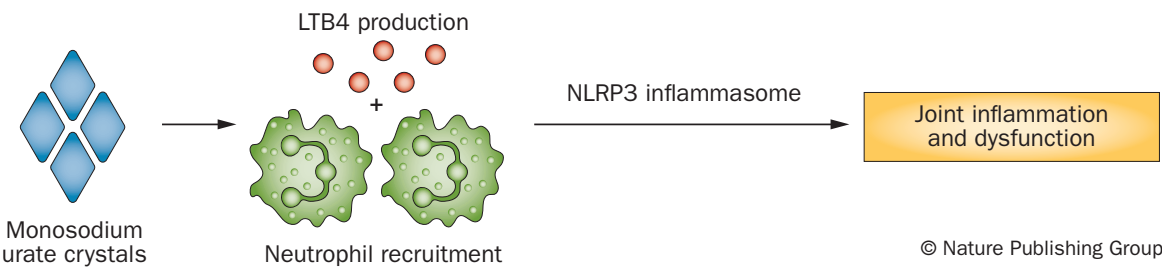

injected them into the knee joints of wild-type mice and mice deficient in inflammatory mediators (such as NLRP3, caspase- 1 and IL-1 $\beta$ ). Inflammation, CXCR2-dependent neutrophil influx and hypernociception were all consequently induced in the wild-type animals; the NLRP3 inflammasome and IL- $1 \beta$ were both critical in mediating these effects. LTB4 levels increased rapidly (within $1 \mathrm{~h}$ after injection) in response to MSU crystals in vivo, which was crucial for caspase-1-dependent IL- $1 \beta$ production and the subsequent release of CXCR2acting chemokines (CXCL1). Moreover, the investigators found that, in vitro,
LTB4 production preceded the cascade of events that led to IL- $1 \beta$ production and NLRP3 activation.

Teixeira notes that more work is need to investigate how these findings translate to humans. "Understanding whether other stimuli (such as asbestos or aluminium hydroxyl salts) also use the LTB4 pathway to trigger the NLRP3 inflammasome is also important," he adds.

\section{Katrina Ray}

Original article Amaral, F. A. et al. NLRP3 inflammasomemediated neutrophil recruitment and hypernociception depends on leukotriene B4 in a murine model of gout. Arthritis Rheum. doi:10.1002/art.33355 